\title{
Infantile hypertrophic pyloric stenosis surgery versus nutritional therapy
}

\begin{abstract}
Objective: to compare the outcomes of surgery versus nutritional therapy in the management of infantile hypertrophic pyloric stenosis (IHPS).

Methods: Thirty-eight IHPS patients were enrolled in the study and subdivided into surgical (22 patients) and nutritional groups (16 patients). Surgery involved extramucosal pyloromyotomy, while nutritional therapy consisted of small and frequent meals only. Patients were monitored both clinically and with ultrasound. A long-term follow-up 13-19years after treatment was carried out to investigate gastric emptying function. Patients were assessed by ultrasound to evaluate gastric volume and emptying time.
\end{abstract}

Results: All 22 surgical patients were cured. One patient from the nutritional group has withdrawn from the study. Nutritional therapy failed in 4/15 patients, who underwent surgery within 48 hours. It was successful in the remaining 11 patients. Pyloric ultrasound parameters returned to normal within 3-4months in both groups of patients. At long-term follow-up of the gastric emptying time was normal in both groups, while a statistically significant increase of antral volume was observed in the surgery group.

Conclusions: Nutritional treatment seemed a viable option in IHPS. Clinical results looked very encouraging even if treatment adoption failed in about $25 \%$ of cases.

Keywords: gastric emptying rate, hypertrophic pyloric stenosis, nutritional therapy, pyloromyotomy
Volume 4 Issue 4 - 2016

\author{
Antonio Dessanti, ${ }^{\prime}$ Vincenzo Migaleddu, ${ }^{2}$ \\ Paolo Castiglia, ${ }^{3}$ Nicola Grandi ${ }^{3}$ \\ 'Pediatric Surgery Unit, University Hospital of Sassari, Italy \\ ${ }^{2}$ Sardinian Mediterranean Imaging Research Group, Italy \\ 3Department of Biomedical Sciences, University Hospital of \\ Sassari, Italy
}

Correspondence: Antonio Dessanti, Pediatric Surgery Unit, University Hospital of Sassari,Via Verona 24- 07100-Sassari (SS)Italy, Tel +393-29|-710-158, Fax 39-079-273469,

Email antoniodessanti@yahoo.it

Received: November 26, 2015 | Published: March 31, 2016
Abbreviations: CG, control group; IHPS, infantile hypertrofic pyloric stenosis; NuG, nutritional group; RP, ramstedt's extramucosal pyloromyotomy; SFM, small and frequent meals; SG, surgical group; US, ultrasound

\section{Introduction}

Infantile hypertrophic pyloric stenosis (IHPS) is a well-known paediatric condition, the pathogeneses of which is still unknown. Several hypotheses on pathogenesis have been suggested over the past decades, involving hormone regulation, genetic and inheritance mutations, molecular and ultrastructural abnormalities. ${ }^{1-7}$ These hypotheses have not modified the treatment of IHPS, which is currently mainly surgical. The surgery involves Ramstedt's extramucosal pyloromyotomy (RP) ${ }^{8}$ This technique releases the pylorus that is compressed by the hypertrophic sphincter muscle to restore gastric emptying. In the past decades medical therapies for IHPS were introduced, mainly based on the pharmacological action of atropine..$^{9-12}$

The aim of this study was to compare the results of the surgical treatment and the nutritional therapy based on small and frequent meals (SFM) in the management of patients with IHPS. During the first year, all patients were monitored clinically and by ultrasound (US) evaluation of the pyloric morphologic parameters and real-time observation of ingested food progression. Preliminary data on these series of patients have already been reported. ${ }^{13} \mathrm{~A}$ long-term follow-up, 13 to $19 y$ years after surgery or nutritional therapy, was performed in order to investigate the gastric emptying functions of these patients. The study was carried out in accordance with the ethical standards of the responsible institutional committee on human experimentation and with the Helsinki Declaration. The Institutional Review Board provided approval for the study.

\section{Materials and methods}

The first part of our study was carried out by analyzing the records of IHPS patients consecutively admitted at the Paediatric Surgery Unit of Sassari University Hospital between 1994 and 2000, with a total number of 38 patients ( 31 males and 7 females). The diagnosis was obtained by clinical and US evidence, according to the morphological pyloric cut-off values: pyloric thickness $>3 \mathrm{~mm}$, length $>15 \mathrm{~mm}$, diameter $>10 \mathrm{~mm}$, volume $>1.5 \mathrm{~cm} 3$, pyloric index $>0.38$ (pyloric volume/weight in kilograms of the infant). ${ }^{14-17}$ All 38 patients were born at term and in good general conditions at birth. At admission they received intravenous hydration for 12 to 48hours, until a good correction of electrolyte imbalance was obtained. Ranitidine therapy was used only when blood traces in the vomited stomach contents were observed. Patients were sequentially allocated to two groups: surgical and nutritional. Sequential allocation to the two treatments guaranteed the casual allocation of patients. In fact, IHPS is not an epidemic event, moreover there is no evidence to hypothesize any possible temporal effect about IHPS severity that could interfere with casual distribution throughout a sequential allocation.

The first 22 patients ( 18 males, 4 females) were surgically treated by conventional RP from 12 to 48 hours after the admission, and included in the surgical group (SG). Feeding was restarted about 6hours after RP with small feeds given over the first 24hours. Afterwards, normal 6-7meals per day were resumed, based on the feeding on demand.

The subsequent 16 IHPS patients (13 males, 3 females) undertook nutritional treatment with small frequent meals only, and were included in the nutritional group $(\mathrm{NuG})$. The nutritional treatment began 6hours after the admission, with 12 small formula meals/day, starting with $10 \mathrm{ml}$. The formula intake was increased by $5 \mathrm{ml}$ every $3-5$ meals until the volume reached $40 \mathrm{ml}$ per meal, within $48-72$ hours. 
The number of meals was then slowly reduced to the normal 6 per day $(120 \mathrm{ml} / \mathrm{kg} /$ day $)$. After each feeding, babies were placed in a halfsitting position. The first 2 days were essential to evaluate if nutritional treatment with SFM could be safely continued: in case of continuous vomiting, the RP was performed within 48hours after admission. A consent form was obtained from the parents of all patients. Special parent's cooperation was necessary from the NuG, since most of the treatment with SFM took place at home under an outpatient supervision programme. Parents of $\mathrm{NuG}$ were informed that some vomiting episodes could occur during the first weeks of nutritional treatment (2-3 episodes per day during the first week were tolerated): in cases of vomiting, they were instructed to give the next scheduled formula intake 15-20minutes after the episode. Demographic data of the two groups of patients are shown in Table 1. For the first year after treatment, all patients were followed-up both clinically and by US. Examinations with US were performed at week 1, 4, 8, 12, and 16 after the operation or from the beginning of the nutritional treatment.

Table I Mean clinical data of surgical and nutritional groups at time of admission. No statistically significant differences were found between means in the study groups (Student T test: $\mathrm{P}>0.05$ )

\begin{tabular}{lll}
\hline Groups & Surgical & Nutritional \\
\hline Number of patients & 22 & $16 *$ \\
Weight at birth & $3.18 \mathrm{Kg}$ & $3.26 \mathrm{Kg}$ \\
Weight at first vomit & $3.75 \mathrm{Kg}$ & $3.91 \mathrm{Kg}$ \\
Weight at admission & $3.6 \mathrm{I} \mathrm{Kg}$ & $3.73 \mathrm{Kg}$ \\
Age at first vomit & 24.73 days & 27.80 days \\
\hline
\end{tabular}

*One patient quit the study three weeks after the beginning of treatment

The statistical significance of the average values differences between the two groups was compared by Hotelling's T2 multivariate analysis. Normality was tested using Shapiro-Wilk test. The second part of this study, consisted of a long-term follow-up examination, carried out 13 to 19years after surgical or nutritional treatment, in order to investigate the patients' gastric function. Our attention was particularly focused on detecting problems related to dyspepsia and gastric emptying. Every endeavour was made to contact the patients from the original groups: reply from $10 \mathrm{SG}$ patients and $10 \mathrm{NuG}$ patient was received. All 20 patients contacted accepted to take part to the proposed long term follow up. Moreover, 10 additional subjects matched by age (same mean age) and gender (same male to female ratio) were enrolled in the study as control group (CG). An informed consent was obtained from each patient or from the parents, if the patients were still under 18years of age.

The 30 patients enrolled were submitted to a clinical examination and a gastric emptying rate exam monitored by US. For this purpose a method of volumetric gastric emptying assessment was designed, according to criteria described by Bolondi et al., ${ }^{18}$ and other studies on gastric emptying rate. A good intraobserver concordance and a good interobserver agreement was demonstrated in the use of real time US in liquid gastric emptying assessments. ${ }^{19,20}$ With a $5 \mathrm{MHz}$ Convex probe, we measured three transversal sections at the level of the pre-pyloric area (S1), angular incisures (S3), and a point between these (S2) (Figure 1). Measurements of each section were performed in triplicate. We obtained three ellipses and calculated an ellipsoid, considering the axes $\mathrm{x}, \mathrm{y}$, and $\mathrm{z}$ of each section using the ultrasound software (Hitachi Aloka ProSound Alpha 7). The antral average volume was obtained considering the three ellipsoid measurements. The patients fasted for 8 hours before the US evaluation. All US tests were performed by the same experienced operator. After a baseline measurement, $400 \mathrm{ml}$ of a fruit juice drink (orange, lemon, and carrot juice: $30 \%$ ) were administrated and $\mathrm{x}, \mathrm{y}$, and $\mathrm{z}$ of the three sections were recalculated after 5,10,20,30, and 40minutes. The curve built on the volume data at different time points allowed gastric emptying time and volume to be assessed. The differences between the SG, $\mathrm{NuG}$, and $\mathrm{CG}$ with respect to the measurements at different times $(0$, $5,10,20,30$, and 40minutes) were evaluated using the analysis of variance for repeated measures. The level of error of the first type was set to a value of probability of $5 \%$. Analyses were performed with STATA software. ${ }^{21}$

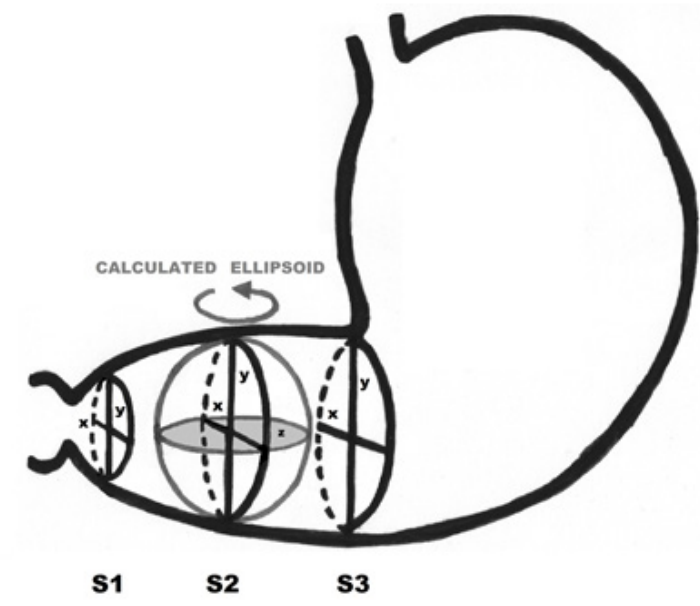

Figure I Volumetric measurement of the antrum.

\section{Results and discussion}

The 22 patients in the SG were clinically cured with RP. One patient in the $\mathrm{NuG}$ was withdrawn from the study three weeks after the beginning of SFM, because of his parents' decision: although nutritional therapy was proceeding well, they stated they were unable to comply with SFM requirements. Parents took the child to another hospital to have surgery. Eleven out of 15 patients in the $\mathrm{NuG}(73.3$ $\%$ ) were clinically cured with a SFM regimen. In these patients vomiting episodes were reported, especially during the first week, with an average of 2 episodes per day. Occasional vomiting episodes were observed over the next three weeks. From the initial 12 small meals/day, the number of meals was reduced to 5 per day over an average of 25days (Figure 2). During the first 2-3days, a slight weight loss was observed in these patients.

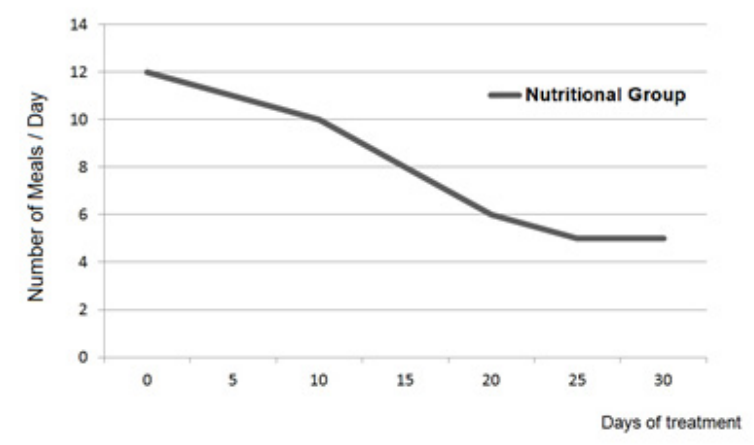

Figure 2 Average number of meals per day during nutritional treatment with small frequent meals.

In four patients belonging to the $\mathrm{NuG}(26.6 \%)$, the SFM therapy failed. They had to have treatment suspended within 48 hours because of persistent and frequent vomiting episodes, which prevented adequate oral nutrition: therefore RP was performed. 
If we compare patients in the $\mathrm{NuG}$, there is a difference between the 11 who were cured with SFM and the 4 who had a deferred surgery (Figure 3). The 11 patients cured had the onset of symptoms at 31.7 \pm 12 .8days of life on average, while the 4 patients who failed had the onset of symptoms at $17 \pm 2.6$ days. This difference was statistically significant $(\mathrm{P}=0.043)$. The same for the weight at admission in hospital, that was on average $3.98 \pm 0.64 \mathrm{Kg}$ for the cured patients against $3.13 \pm 0.22 \mathrm{Kg}$ for the patients who failed the SFM therapy $(\mathrm{P}=0.023)$.

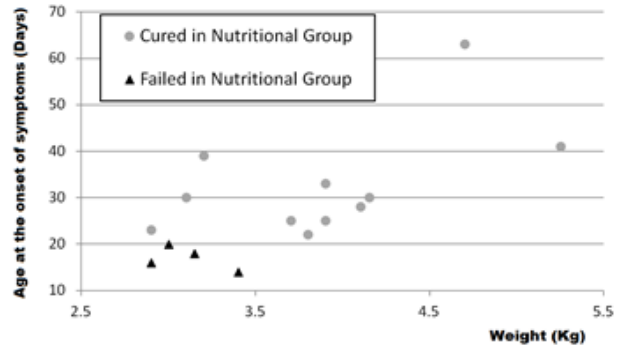

Figure 3 Age at the onset of symptoms and weight at time of admission of patients of the nutritional group.

In six patients from the $\mathrm{SG}$ and in 4 from the $\mathrm{NuG}$, for which occasional blood traces in vomited milk in their emesis were reported, therapy with ranitidine for 7days was administered for suspected gastritis and/or reflux esophagitis. The length of stay in hospital was on average 5 days for $\mathrm{SG}$ patients and 4 days for $\mathrm{NuG}$ patients. Two patients in NuG were treated in outpatients clinic. Analyzing our outcomes from an economical point of view the direct cost for SG was 5.161€ (7.171 USD) per patient, while direct cost for $\mathrm{NuG}$ was $1.519 €$ (2.066 USD) per patient. Costs were calculated based on Diagnosis Related Groups in our district. The follow-up during the first year showed that all patients were healthy and they were growing normally. It was relevant that none of the $11 \mathrm{NuG}$ patients reported problems related to gastric emptying.

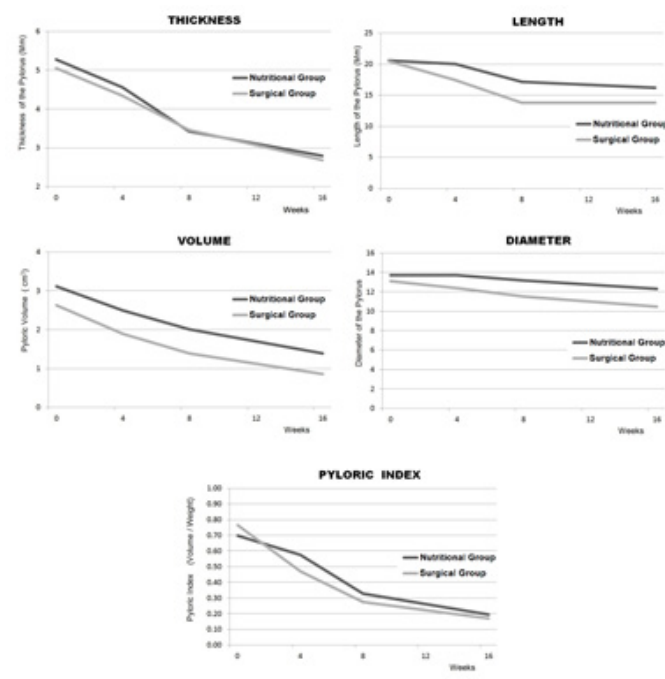

Figure 4 Morphological pyloric US parameters monitored during the followup. The parameters normalize over about 12 weeks in both groups.

The course of the infant's weight gain after treatment was similar in SG patients and the NuG patients. In all patients the pyloric US parameters returned to normal within 3-4months (Figure 4). Although this seemed to happen slightly faster in the SG, the difference between the two groups was not statistically significant. All patients included in the long-term follow-up (10 from the SG, 10 from the $\mathrm{NuG}$, and 10 $\mathrm{CG})$ were in good health at the time of the examination and none of them reported problems related to gastric emptying in the anamnesis.

The US evaluation of gastric emptying showed no statistically significant differences between the three groups if we consider the gastric emptying time $(P=0.46)$. A significant difference $(P<0.001)$ was observed between the three groups in the curves for antral volume at different times of the measurement. In fact, the average antral volumes were similar in the $\mathrm{CG}$ and $\mathrm{NuG}(\mathrm{P}=0.71)$, while an increase in the antral volume was observed in the $\mathrm{SG}$ ( $\mathrm{SG}$ vs $\mathrm{NuG}$ : $\mathrm{P}<0.001$; SG vs CG: $\mathrm{P}<0.001$ ) (Figure 5). Surgical and conservative treatment in IHPS is still under debate and worthy of attention. When we analyzed the US evidence on the hypertrophic pylorus after surgical or nutritional treatment, we observed its progressive normalization over 12 weeks approximately. ${ }^{14,16,17}$ This observation, confirmed by data from our study, reinforces the theory that describes IHPS as a self-healing condition.

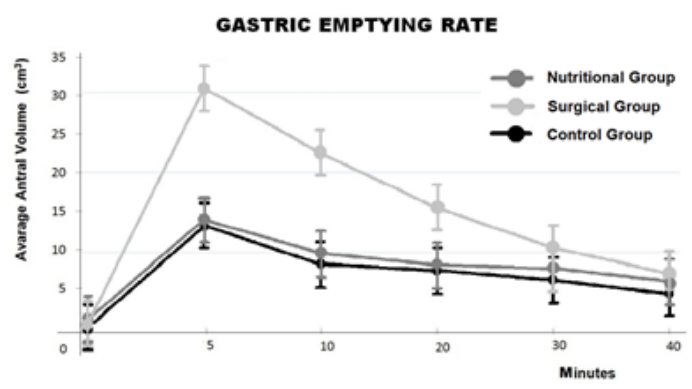

Figure 5 Gastric emptying examination by ultrasounds. The total emptying time is similar among all three groups. A significant increase of the antral volume is detectable in the surgical group when compared to the nutritional group and the control group.

The RP does not heal the pylorus, but restores the transit, allowing gastric emptying. The surgery gives to the infant the necessary time for the pylorus to restore normal functions. Based on the concept that IHPS is a disease that mysteriously appears then regresses, the objective of the nutritional therapy should be to achieve a rapid oral refeeding in the same way as the surgical treatment does. Unlike the previous medical studies based on atropine action, in the present study we adopted the nutritional therapy with SFM alone. The main reason for this therapeutic choice was the fact that a little pyloric transit is retained in IHPS. Radiographic examinations with barium meal and more recently US have always emphasized this aspect. The second reason is that SFM do not cause excessive dilatation of the stomach, consequently the stomach is less stimulated to contract, thus breaking the vicious circle represented by pyloric hypertrophy, delayed gastric emptying, gastric dilatation, gastric contraction and projectile vomiting. The results of our study showed that SFM treatment was successful in about $70 \%$ of the infants. In 1946, Jacoby ${ }^{9}$ introduced some useful criteria to distinguish infants eligible for conservative treatment (drug therapy with atropine sulfate and small feedings) from the infants who should have had immediate surgery. For Jacoby, hematemesis, severe dehydration, or the onset of symptoms before the second week of life were indications for elective surgery. Medical treatment was given to children who had symptoms during or after the fourth week of life and in which dehydration was mild. Analyzing our data in comparison to Jacoby's observations, we found that 4 patients of the $\mathrm{NuG}$ who required deferred surgery had an earlier onset of symptoms ( $17 \pm 2.58$ days of life) and a lower body weight $(3.11 \pm 0.22 \mathrm{Kg})$ at the time of admission. On the basis of these results, we hypothesize that body weight at admission and the age at onset of 
symptoms may predict the probability of failure of SFM therapy. In fact, since we observed a modest loss of weight during the first days of nutritional treatment, this phenomenon may interact with the low weight of the newborns at an early onset of symptoms, leaving them with fewer caloric reserves. On the other hand, the number of failures in this experience is too low to be conclusive. Additional studies are needed to help clarify this hypothesis.

The results of our long-term follow-up study with US showed no significant differences in total gastric emptying time among the $\mathrm{SG}, \mathrm{NuG}$, and CG. However, considering the volumes of the antrum at different times of observation, we found differences among the 3 groups. While the curves for the NuG and CG showed similar volumes, the gastric emptying curve for all patients in the SG showed an antral volume increase of $80 \%$, compared to the $\mathrm{NuG}$ and $\mathrm{CG}$ group (Figure 5). Although it is not related to any kind of clinical symptom, it is reasonable to believe that cutting the circular fibers in the pyloric sphincter muscle during RP might cause some alterations in the delicate mechanisms that regulate the gastric emptying. This may lead to hypothesize that the pylorus after RP requires a greater volume or an increased pressure on the gastric antrum to be opened because it seems more rigid or partially denervated. These functional consequences over the years should be further analyzed.

\section{Conclusion}

Nutritional treatment seemed a viable option in IHPS. Clinical results looked very encouraging even if treatment adoption failed in about $25 \%$ of cases. However, this means that the remaining $75 \%$ affected by IHPS can be cured through a nutritional therapy. This can be crucial if patients affected are born in a country with limited resources, where surgical paediatric units are not available. Moreover, it has to be mentioned that Ramsted's pyloromyotomy is not free from complications, including severe complications, in $5 \%$ of cases ${ }^{22}$. We believe that nutritional treatment might be considered as an alternative to surgery in infants with IHPS, especially in children who present the onset of symptoms after the third week of life and consequently a higher body weight at admission.

\section{Acknowledgements}

The authors are especially grateful to Doctor Kathleen Schwarz, (Johns Hopkins University, Director of the Pediatric Liver Center) for her thoughtful and helpful review of this manuscript.

\section{Conflicts of interest}

Author declares there are no conflicts of interest.

\section{Funding}

None.

\section{References}

1. Panteli C. New insights the pathogenesis of infantile pyloric stenosis Pediatr Surg Int. 2009;25(12):1043-1052.

2. Dick AC, Ardill J, Potts SR, et al. Gastrin, somatostatin and infantile hypertrophic pyloric stenosis. Acta Paediatr. 2001;90(8):879-882.

3. Capon F, Reece A, Ravindrarajah R, et al. Linkage of monogenic infantile hypertrophic pyloric stenosis to chromosome 16p12-p13 and evidence for genetic heterogeneity. Am J Hum Genet . 2006;79(2):378-382.

4. Everett KV, Chioza BA, Geourgoula C, et al. Genome-wide high-density SNP-based linkage analysis of infantile hypertrophic pyloric stenosis identifies loci on chromosomes 11q14-q22 and Xq23. Am J Hum Genet . 2008;82(3):756-762.

5. Serra A, Schuchardt K, Genuneit J, et al. Genomic variants in the coding region of neuronal nitric oxide synthase (NOS1) in infantile hypertrophic pyloric stenosis. J Pediatr Surg. 2011; 46(10):1903-1908.

6. Feenstra B, Geller F, Krogh C, et al. Common variants near MBNL1 and NKX2-5 are associated with infantile hypertrophic pyloric stenosis. Nat Genet. 2012; 44(3):334-337.

7. Guarino N, Shima H, Puri P. Structural immaturity of the pylorus muscle in infantile hypertrophic pyloric stenosis. Pediatr Surg Int. 2000;16(4):282-284.

8. Ramsted C. Zur operation der angenboreben pylorus stenose. Med Klin. 1912; 8:1702-1705.

9. Jacoby NM. Pyloric stenosis: selective medical and surgical treatment. Br Med J . 1946;1(4453):721-722.

10. Nagita A, Yamaguchi J, Amemoto K, et al. Management and ultrasonographic appearance of infantile hypertrophic pyloric stenosis with intravenous atropine sulfate. J Pediatr Gastroenterol Nutr. 1996;23(2):172-177.

11. Kawahara H, Imura $\mathrm{K}$, Nishikawa $\mathrm{M}$, et al. Intravenous atropine treatment in infantile hypertrophic pyloric stenosis. Arch Dis Child. 2002;87(1):71-74.

12. Kawahara H, Takama Y, Yoshida H, et al. Medical treatment of infantile hypertrophic pyloric stenosis: should we always slice the "olive"? $J$ Pediatr Surg . 2005;40(12):1848-1851.

13. Migaleddu V, Dessanti A, Castiglia P, et al. US of hypertrophic pyloric stenosis: morphologic and volumetric changes of the pyloric olive after surgery versus conservative therapy. Radiology supplements. $1995 ; 197: 159$

14. Carver RA, Okorie NM, Steiner GM, et al. Infantile hypertrophic pyloric stenosis-diagnosis from the pyloric muscle index. Clin Radiol. 1987;38(6):625-627.

15. Davies PR, Linke RJ, Robinson RG, et al. Sonographic diagnosis of infantile hypertrophic pyloric stenosis. $J$ Ultrasound Med.1 992;11(11):603-605.

16. Muramori K, Nagasaki A, Kawanami T. Ultrasonographic serial measurements of the morphologic resolution of the pylorus after Ramstedt pyloromyotomy for infantile hypertrophic pyloric stenosis. $J$ Ultrasound Med. 2007;26(12):1681-1687.

17. Huang YL, Lee HC, Yeung CY, et al. Sonogram before and after pyloromyotomy: the pyloric ratio in infantile hypertrofic pyloric stenosis. Pediatr Neonatol . 2009;50(3):117-120.

18. Bolondi L, Bortolotti M, Santi V, et al. Measurement of gastric emptying time by real-time ultrasonography. Gastroenterology. 1985;89(4): 752-759.

19. Irvine EJ, Tougas G, Lappalainen R, et al. Reliability and interobserver variability of ultrasonographic measurement of gastric emptying rate. Dig Dis Sci . 1993;38(5):803-810.

20. Asai A, Takehara H, Harada M, et al. Ultrasonographic evaluation of gastric emptying in normal children and children after pyloromyotomy. Pediatr Surg Int . 1997;12(5-6): 344-347.

21. StataCorp. Stata Statistical Software: Release 12.1 College Station, TX: Stata Corp LP. 2011.

22. Perger L, Fuchs JR, Komidar L, et al. Impact of surgical approach on outcome in 622 consecutive pyloromyotomies at a pediatric teaching institution. J Pediatr Surg . 2009;44(11):2119-2125. 